\title{
Rapid Quantification of Bioactive Lentinan with an Aniline Blue Fluorescent Method
}

\author{
Luodi Fan', Tong $\mathrm{Li}^{2}$, Minghua $\mathrm{Hu}^{1}$, Fangli Ma1, Boyang $\mathrm{Yu}^{2 *}$, Jiangwei Tian ${ }^{2 *}$ \\ ${ }^{1}$ Infinitus (China) Company Ltd., Guangzhou, China \\ ${ }^{2}$ Jiangsu Key Laboratory of TCM Evaluation and Translational Research, School of Traditional Chinese Pharmacy, China \\ Pharmaceutical University, Nanjing, China \\ Email: *boyangyu59@163.com, *jwtian@cpu.edu.cn
}

How to cite this paper: Fan, L.D., Li, T., Hu, M.H., Ma, F.L., Yu, B.Y. and Tian, J.W. (2019) Rapid Quantification of Bioactive Lentinan with an Aniline Blue Fluorescent Method. Pharmacology \& Pharmacy, 10, 318-328.

https://doi.org/10.4236/pp.2019.106026

Received: May 20, 2019

Accepted: June 25, 2019

Published: June 28, 2019

Copyright (C) 2019 by author(s) and Scientific Research Publishing Inc. This work is licensed under the Creative Commons Attribution International License (CC BY 4.0).

http://creativecommons.org/licenses/by/4.0/

\begin{abstract}
Lentinan is a clinically approved immune modulator and its anticancer and immunomodulatory bioactivity is found to be dependent on its triple helical conformation. Therefore, the development of rapid and convenient method for determination of bioactive lentinan with triple helical conformation holds great promise for the quality control of lentinan healthy products. In this work, an aniline blue fluorescent method was optimized and established to accurately and rapidly detect bioactive lentinan. In the presence of lentinan, the fluorescence intensity of aniline blue with $404 \mathrm{~nm}$ excitation and $492 \mathrm{~nm}$ emission dramatically enhanced within $15 \mathrm{~min}$ in $\mathrm{pH} 10$ glycine- $\mathrm{NaOH}$ buffer solution, which allowed the analysis of lentinan in a very simple and fast manner. The method allowed for the sensitive determination of lentinan in the range of 1 to $60 \mu \mathrm{g} / \mathrm{mL}$ with a detection limit of $0.25 \mu \mathrm{g} / \mathrm{mL}$. Notably, the protocol exhibited excellent selectivity for the determination of triple helical lentinan over other saccharides. The method was successfully applied to the detection of bioactive lentinan in health tonic solution, which demonstrated the method had great potential for quality control of lentinan contained products.
\end{abstract}

\section{Keywords}

Lentinan, Triple Helical Conformation, Aniline Blue, Fluorescence Detection, Health Tonic Solution

\section{Introduction}

Lentinan is isolated from the fruiting body of Lentinus edodes (Shiitake) [1] [2] and is known as a bioactive polysaccharide due to its strong antitumor activity through the activation of human immune system [1] [3] [4] [5] [6]. Lentinan has been approved as an immune modulator for tumor treatment and clinically ap- 
plied in combination with chemotherapy [7] [8]. With greater emphasis on the prevention of diseases, consumption of health products containing lentinan has grown in popularity. Recent researches have demonstrated that the immunotherapeutic activity of lentinan is mainly dependent on the polysaccharide's junior and senior structures [9] [10]. Lentinan is a $\beta$-glucan with the repeating unit consisting of two $\beta$-(1,6)-glucopyranoside branches for every five linear $\beta$-(l,3)glucopyranoside linkages [11] [12] [13] [14]. Owing to the inter and intra molecular hydrogen bonds, lentinan can form the helical structures especially triple helical conformation in aqueous solution [15], which plays critical role in the immunoregulatory function [16] [17] [18]. Therefore, developing a rapid and convenient method for quantification of triple helical lentinan is highly desired to evaluate the quality of lentinan containing healthy products.

At present, there are mainly three kinds of methods to measure $\beta$-glucan content, including inorganic chemistry-based method [19] [20], enzymic method [21], and protein specific identification method [22] [23]. The first two kinds of methods utilize acid or exoglucanase to hydrolyze the $\beta$-glucan into glucose. The content of $\beta$-glucan can be calculated by determination of the generated glucose concentration. Although the detecting sensitivity is high, these two methods suffer unsatisfactory specificity because they can only detect the total glucose content and may obtain higher results than the actual value in the presence of other polysaccharide such as starch [21]. Moreover, these methods are unable to directly measure the content of the triple helical lentinan. Protein specific identification method like enzyme-linked immunosorbent assay [22].and limulus factor $\mathrm{G}$ test [24] that uses specific protease and polyclonal antibody for $\beta$-glucan detection can meet the requirements of specificity. However, the method is costly and time-consuming. Therefore, there is still a strong need to develop a sensitive, specific, rapid and cost-efficient detection method for helical lentinan.

The fluorimetry method is a powerful tool due to its high sensitivity and simplicity in data collection [24] [25]. In this work, a fluorimetry method using aniline blue as the probe has been established to detect helical lentinan. Aniline blue, with the structure of sodium 4,4'-[carbonylbis-(benzene-4,1-diyl)bis-(imno)] bis(benzene-sulphonate) [26] [27], is a water-soluble weakly fluorescent dye. Upon binding to the triple helical lentinan, the formed complex of aniline blue/ lentinan produces strong fluorescence, which is potential and promising for quantitation of bioactive lentinan in health products. Therefore, in this study, we have optimized an aniline blue fluorescent method by investigating a variety of impact factors in fluorescence intensity of the aniline blue/lentinan complex. Furthermore, this fluorimetry method has been employed for direct detection of bioactive lentinan content in oral health tonic liquid.

\section{Materials and Methods}

\subsection{Materials and Reagents}

Lentinan with an average molecular weight of $630,000 \mathrm{Da}$ (purity $>98 \%$ ) was 
purchased from Yuanfu Pharmaceutical Science and Technology Co. Ltd. (Shanghai, China). Aniline blue was purchased from Nanjing Wanqing Chemical Glassware Instrument Co. Ltd. (Nanjing, China). Glucose, sucrose, maltose and starch were purchased from Sinopharm Chemical Reagent Co. Ltd. (Nanjing, China). Xylan and xanthan gum were purchased from Aladdin Industrial Co. Ltd. (Shanghai, China). Oral health tonic liquid was supplied by Infinitus Co. Ltd. (Guangzhou, China). All reagents were of at least analytical grade and used without further purification. All solutions were prepared with ultrapure water (18.2 M $\Omega$ ) purified by a Millipore Simplicity System (Millipore, Bedford, USA).

\subsection{Apparatus}

Fluorescence intensity and spectra were recorded by Varioskan Flash Multimode Reader (Thermo Scientific). pH meter (Delta 320) was applied to measure the $\mathrm{pH}$ values of solutions.

\subsection{Reagents Preparation}

A series of glycine- $\mathrm{NaOH}$ buffer solutions with different $\mathrm{pH}$ values were prepared by mixing $0.2 \mathrm{~mol} / \mathrm{L}$ glycine with $\mathrm{NaOH}$ or $\mathrm{HCl}$ solutions at different proportions, and the $\mathrm{pH}$ values were adjusted by $\mathrm{pH}$ meter. $100 \mu \mathrm{g} / \mathrm{mL}$ lentinan stock solution was prepared by dissolving $0.0100 \mathrm{~g}$ of lentinan in a $0.5 \mathrm{~mol} / \mathrm{L}$ $\mathrm{NaOH}$ solution and adjusted to $\mathrm{pH} 10$ with $0.2 \mathrm{~mol} / \mathrm{L}$ glycine, and then diluted with $0.1 \mathrm{~mol} / \mathrm{L}$ glycine- $\mathrm{NaOH}$ buffer solution in a $100 \mathrm{~mL}$ volumetric flask. The lentinan stock solution was stored in $4^{\circ} \mathrm{C} .0 .1 \%(\mathrm{w} / \mathrm{v})$ of aniline blue stock solution was prepared by dissolving $0.1000 \mathrm{~g}$ of aniline blue with $0.1 \mathrm{~mol} / \mathrm{L}$ glycine- $\mathrm{NaOH}$ buffer in a $100 \mathrm{~mL}$ volumetric flask and stored in dark to preserve it from light.

\subsection{Fluorescence Determination of Lentinan}

Different volumes $(0-6 \mathrm{~mL})$ of lentinan stock solution were mixed with $4 \mathrm{~mL}$ aniline blue solution $(0.1 \%)$, and the mixtures were diluted to $10 \mathrm{~mL}$ with $\mathrm{pH} 10$ glycine- $\mathrm{NaOH}$ buffer solution in brown volumetric flasks. The resulted solutions were heated at $80^{\circ} \mathrm{C}$ for $15 \mathrm{~min}$ and then gradually cooled to room temperature. Afterwards $100 \mu \mathrm{L}$ mixture per well was added into the 96-well black plate, and the fluorescence intensity was measured by Varioskan Flash Multimode Reader. The samples were excited at $404 \mathrm{~nm}$ and the emission was collected at $492 \mathrm{~nm}$. The calibration curve of lentinan was constructed by plotting the relative fluorescence intensity against the corresponding lentinan concentration using linear regression model. The limit of detection (LOD) was determined as the concentration of the lentinan in the solution when the signal to noise ratio $(\mathrm{S} / \mathrm{N})$ was approximately 3 .

\subsection{Specificity Assay}

$0.4 \mathrm{~mL}$ lentinan stock solution was mixed with different volumes $(0,0.2,0.4,0.6$, 
$0.8,1,1.2,1.4,1.6,1.8$, and $2.0 \mathrm{~mL}$ ) of $1 \mathrm{mg} / \mathrm{mL}$ other potentially interferential polysaccharides including glucose, sucrose, maltose, starch, cellulose, xanthan gum, and xylan. Then $4 \mathrm{~mL} \mathrm{1 \%}$ aniline blue stock solution was added, and the mixture was diluted to $10 \mathrm{~mL}$ with $\mathrm{pH} 10$ glycine- $\mathrm{NaOH}$ buffer solution. The resulted solution was heated at $80^{\circ} \mathrm{C}$ for $15 \mathrm{~min}$ and gradually cooled to room temperature to perform fluorescence assay. In addition, dimethyl sulfoxide (DMSO) was added to investigate the specific binding between aniline blue and the lentinan with triple helical conformation. $1.0 \mathrm{mg}$ lentinan was dissolved in different volumes of DMSO and diluted to $10 \mathrm{~mL}$ using $\mathrm{pH} 10$ glycine- $\mathrm{NaOH}$ buffer solution with the DMSO concentration of $0,2 \%, 10 \%$, and $50 \%$, respectively. Taken $0.4 \mathrm{~mL}$ solution into $4 \mathrm{~mL} 0.1 \%$ aniline blue stock solution, and the mixture was diluted to $10 \mathrm{~mL}$ with $\mathrm{pH} 10$ glycine- $\mathrm{NaOH}$ buffer solution to perform fluorescence assay.

\subsection{Fluorescence Detection of Lentinan in Health Tonic Solution}

Different volumes $(0,0.25,0.50$ and $0.75 \mathrm{~mL})$ of $0.1 \mathrm{mg} / \mathrm{mL}$ lentinan solution were mixed with $0.25 \mathrm{~mL}$ health tonic solution, and then $4 \mathrm{~mL} 0.1 \%$ aniline blue stock solution was added. The solution was diluted to $10 \mathrm{~mL}$ with $\mathrm{pH} 10$ glycine- $\mathrm{NaOH}$ buffer solution. The established fluorescence method was performed to obtain the recovery to evaluate the accuracy of the method.

\section{Results and Discussion}

\subsection{Fluorescence Spectra of Aniline Blue Fluorescent Method}

Fluorescence spectra of aniline blue in the absence and presence of lentinan were measured to investigate the feasibility of aniline blue fluorescent method to detect lentinan. As shown in Figure 1, the excitation and emission of aniline blue was very weak in aqueous solution, which could be attributed to the intramolecular C-C rotation of aniline blue. After addition of lentinan, strong fluorescence

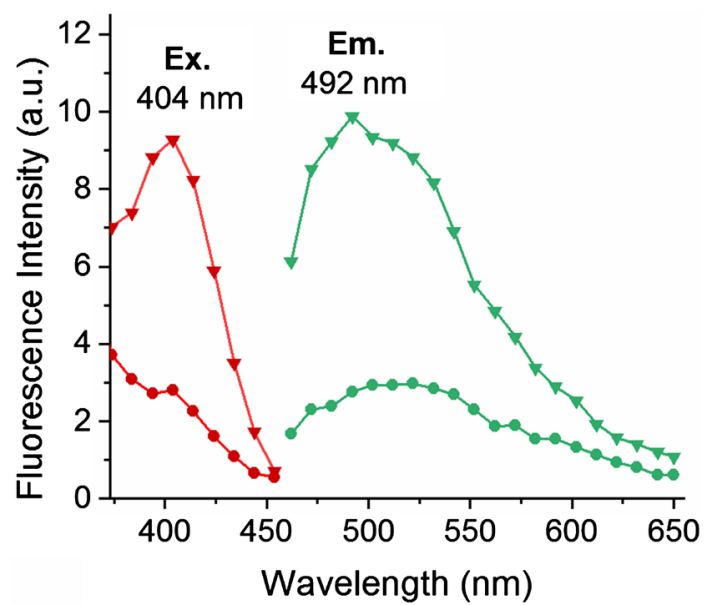

Figure 1. Fluorescence excitation (red line) and emission (green line) spectra of aniline blue in the absence (dotted line) and presence (trigonal line) of $5 \mu \mathrm{g} / \mathrm{mL}$ lentinan in $0.1 \mathrm{M}$ $\mathrm{pH} 10.0$ glycine- $\mathrm{NaOH}$ buffer. 
enhancement was observed which indicated the formation of aniline blue/lentinan complex. The result suggested that upon binding to the cavity of triple helical structure of lentinan, the intramolecular C-C rotation of aniline blue was inhibited which increases the coplanarity and leaded to an enhanced fluorescence for lentinan detection. For the following experiments, $404 \mathrm{~nm}$ and $492 \mathrm{~nm}$ were chosen as the excitation and emission wavelength, respectively.

\subsection{Optimization of Detection Conditions}

Several detection conditions of the aniline blue fluorescent method that may affect the assay of lentinan were investigated, including the concentration of aniline blue stock solution, incubation $\mathrm{pH}$, temperature, and time. When the concentration of aniline blue stock solution was lower than $0.1 \%(\mathrm{w} / \mathrm{v})$, the fluorescence intensity of aniline blue/lentinan complex increased along with the increasing concentration of aniline blue (Figure 2(a)). When the concentration was higher than $0.1 \%(\mathrm{w} / \mathrm{v})$, the fluorescence intensity decreased sharply, which may due to the self-quenching effect of aniline blue molecules. Thus, a concentration

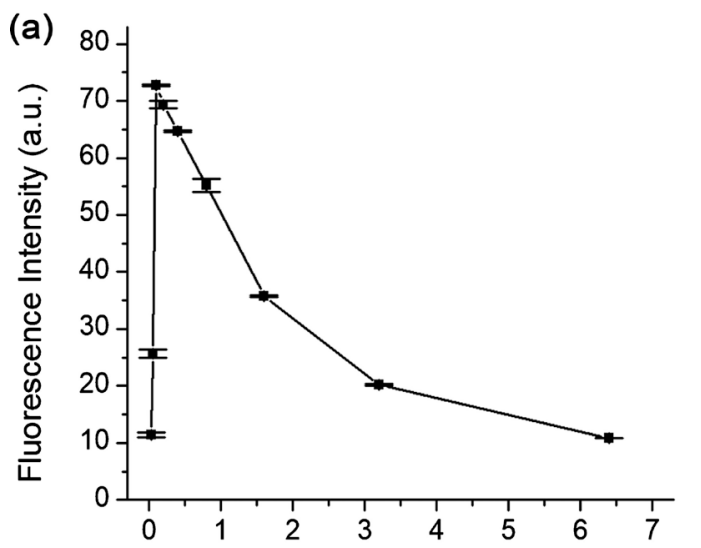

Concentration of aniline blue stock solution (\%)

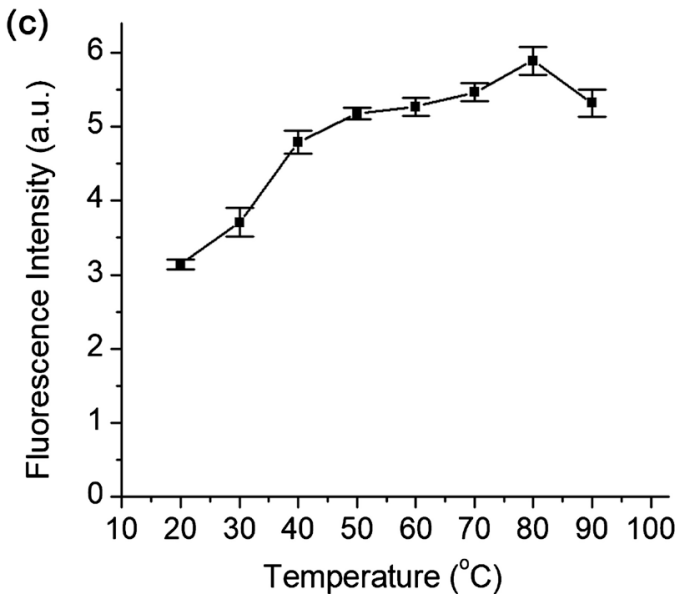

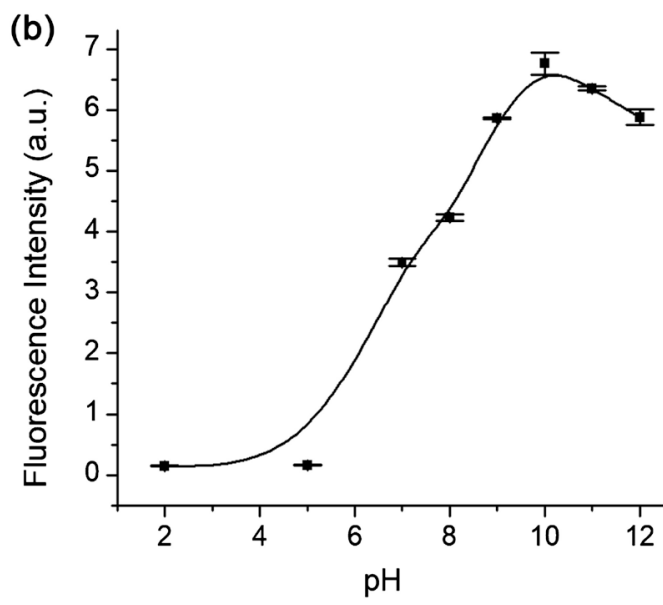

(d)

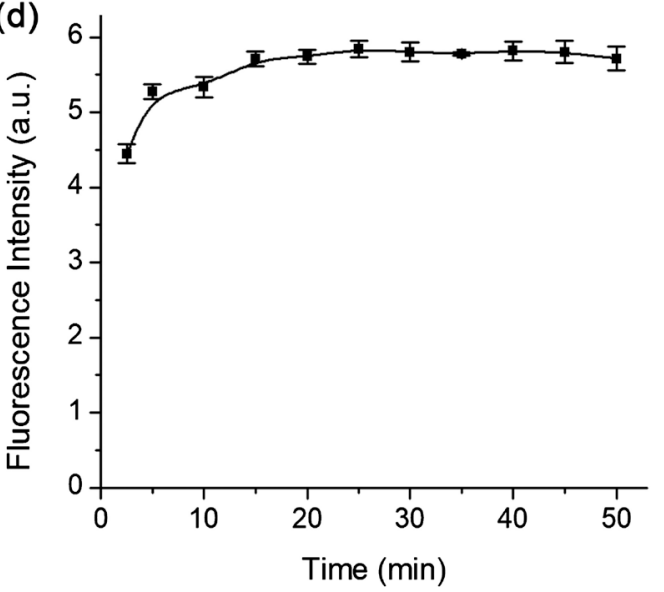

Figure 2. (a) Fluorescence intensity of aniline blue $\left(\lambda_{\mathrm{ex}}=404 \mathrm{~nm}, \lambda_{\mathrm{em}}=492 \mathrm{~nm}\right)$ with various concentrations of aniline blue stock solution from $0.025 \%$ to $6.4 \%(\mathrm{w} / \mathrm{v})$ in the presence of $60 \mu \mathrm{g} / \mathrm{mL}$ lentinan. Effects of (b) $\mathrm{pH},(\mathrm{c})$ temperature, and (d) incubation time on the fluorescence intensity $\left(\lambda_{\mathrm{ex}}=404 \mathrm{~nm}, \lambda_{\mathrm{em}}=492 \mathrm{~nm}\right)$ of aniline blue in the presence of $4 \mu \mathrm{g} / \mathrm{mL}$ lentinan in glycine- $\mathrm{NaOH}$ buffer. Data are means $\pm \mathrm{SD}(\mathrm{n}=3)$. 
of $0.1 \%(\mathrm{w} / \mathrm{v})$ aniline blue stock solution was selected for the following experiments.

The effect of $\mathrm{pH}$ on the fluorescence intensity of aniline blue/lentinan complex was also investigated. The fluorescence intensity was observed in glycine- $\mathrm{NaOH}$ buffer solution with the $\mathrm{pH}$ ranging from 2 to 12 (Figure 2(b)). The results showed that the fluorescence intensity was very weak under weakly acidic medium. This observation indicated that the acidic $\mathrm{pH}$ could influence the intraand inter-molecular hydrogen bonds of lentinan, which damaged the triple helical structure of lentinan [28] and inhibited the formation of aniline blue/lentinan complex. However, the fluorescence intensity increased conspicuously with the $\mathrm{pH}$ from 6 to 12, and the intensity reached a maximum value at $\mathrm{pH} 10$ which was selected as the optimum incubation $\mathrm{pH}$.

The effect of incubation temperature from $20^{\circ} \mathrm{C}$ to $90^{\circ} \mathrm{C}$ on the fluorescence intensity was examined (Figure $2(\mathrm{c})$ ). The fluorescence intensity increased gradually along with the temperature from $20^{\circ} \mathrm{C}$ to $80^{\circ} \mathrm{C}$, suggesting that higher temperature could prompt the molecular motion of aniline blue to bind with lentinan. While the temperature was higher than $80^{\circ} \mathrm{C}$, the fluorescence intensity unexpectedly declined, indicating that excessive temperature may lead to the nonradiative transition probability of aniline blue molecules. For the following experiments, $80^{\circ} \mathrm{C}$ was chosen as the optimum reaction temperature.

The influence of time was also investigated. The fluorescence intensity increased rapidly within $10 \mathrm{~min}$ (Figure $2(\mathrm{~d})$ ). After $15 \mathrm{~min}$, it remained nearly constant, indicating a fast binding between aniline blue and lentinan. Therefore, an optional incubation time of $15 \mathrm{~min}$ was selected to ensure a rapid detection of lentinan.

\subsection{Sensitivity and Selectivity for Lentinan Detection}

To evaluate the sensitivity of this aniline blue fluorescent approach for quantitative analysis under the optional conditions, the assay was practiced at different amounts of lentinan. As shown in Figure 3, a good linear relationship between relative fluorescence intensity and lentinan concentrations was obtained over the range from 1 to $60 \mu \mathrm{g} / \mathrm{mL}\left(\mathrm{R}^{2}=0.9997\right)$. The LOD (the concentration when $\mathrm{S} / \mathrm{N}$ was approximately 3) was determined to be as low as $0.25 \mu \mathrm{g} / \mathrm{mL}$, demonstrating the high sensitivity of the aniline blue fluorescent method.

Discrimination of lentinan from a large excess of other saccharides is a critical issue for lentinan detection. We examined the selectivity of the aniline blue fluorescence method for lentinan by detecting the response of lentinan in the presence of potentially interfering saccharides, including glucose, sucrose, maltose, cellulose, starch, xanthan gum, and xylan. The results showed that almost no fluorescence change was observed for the addition of those saccharides (Figure 4 ), suggesting the presence of these interferences did not affect the quantitative determination of lentinan. Furthermore, DMSO as a powerful hydrogen bond acceptor was added to break the triple helical structure of lentinan to be coil conformation [9] [29] [30]. With the increasing concentration of DMSO from 0 to $50 \%$, the fluorescence intensity decreased significantly (Figure 5), demonstrating 


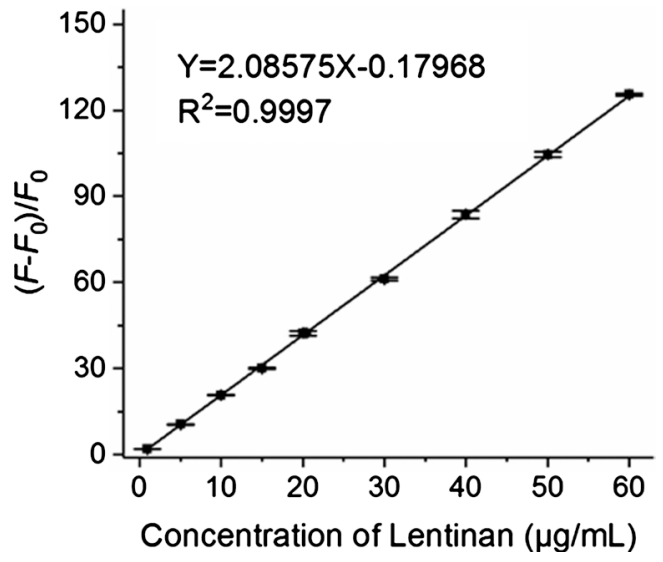

Figure 3. Relative fluorescence intensity $\left(\lambda_{\mathrm{ex}}=404 \mathrm{~nm}, \lambda_{\mathrm{em}}=492 \mathrm{~nm}\right)$ of aniline blue in the presence of increasing concentration of lentinan from 1 to $60 \mu \mathrm{g} / \mathrm{mL}$. $F$ and $F_{0}$ are fluorescence intensities in the presence and absence of lentinan, respectively. Data are means \pm SD $(n=3)$.

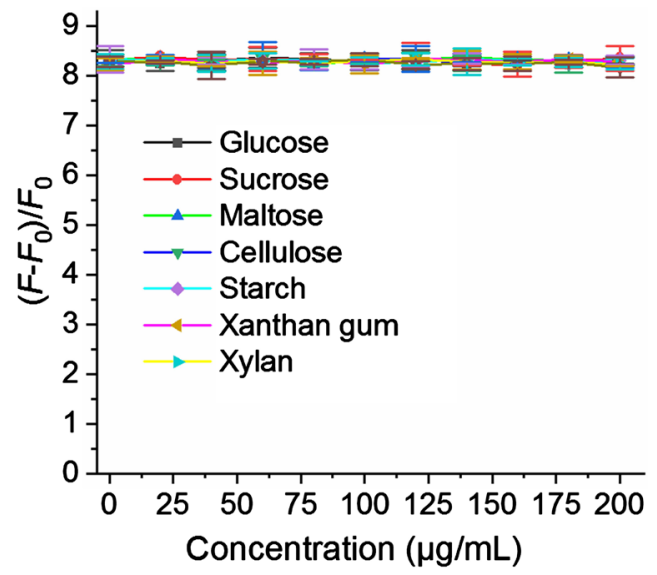

Figure 4. Relative fluorescence response $\left(\lambda_{\mathrm{ex}}=404 \mathrm{~nm}, \lambda_{\mathrm{em}}=492 \mathrm{~nm}\right)$ of aniline blue to 4 $\mu \mathrm{g} / \mathrm{mL}$ lentinan in the presence of various saccharides with the concentration from 0 to $200 \mu \mathrm{g} / \mathrm{mL}$. Data are means $\pm \mathrm{SD}(\mathrm{n}=3)$.

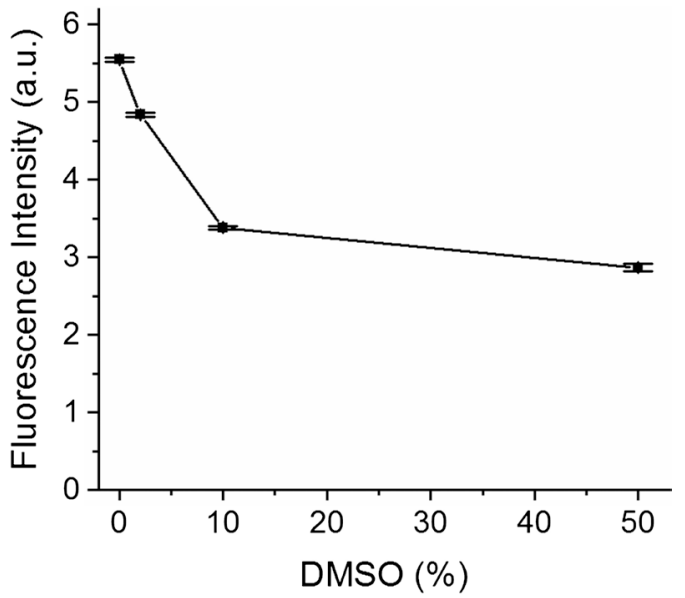

Figure 5. Effect of DMSO on the fluorescence intensity $\left(\lambda_{\mathrm{ex}}=404 \mathrm{~nm}, \lambda_{\mathrm{em}}=492 \mathrm{~nm}\right)$ of aniline blue in the presence of $4 \mu \mathrm{g} / \mathrm{mL}$ lentinan in $\mathrm{pH} 10.0$ glycine- $\mathrm{NaOH}$ buffer. Data are means $\pm \operatorname{SD}(\mathrm{n}=3)$. 
the specificity of binding between aniline blue and triple helical structure of lentinan. Glucose, sucrose, maltose, and cellulose were dissociative in aqueous solution without helical structure. The conformations of starch and xanthan gum were single helix and double helix, respectively. Thus, aniline blue could not bind to the cavity of the single or double helical structure. Although xylan possessed triple helical conformation, the glucan was not $\beta-(1,3)-(1,6)$. Therefore, the excellent selectivity could be attributed to the specificity of complementary structure between aniline blue and the unique triple helical structure formed by $\beta$-(1,3)-(1,6)-glucan of lentinan in aqueous solution, which ensured the selectivity of this approach for lentinan products determination.

\subsection{Detecting Lentinan in Health Tonic Solution}

In order to examine the performance of this method for practical applications, the recovery test in health tonic solution was conducted. The recovery and RSD were analyzed by the standard addition method, and the results were listed in Table 1 . The recovery was $98.9 \%-103 \%$ with an average recovery rate of $101 \%$. The relative standard deviation was $1.73 \%$. These results indicated that the presence of lentinan in health tonic solution could be precisely detected. Furthermore, aniline blue fluorescent method and phenol-sulfuric acid method as a common measurement were used to determine the original lentinan concentration in 10 samples of health tonic solution, respectively. The results showed that the concentrations of lentinan determined by aniline blue method were 197 236 $\mu \mathrm{g} / \mathrm{mL}$ with the average concentration of $216 \mu \mathrm{g} / \mathrm{mL}$ (Table S1 in Supporting Information). As a control group, the concentrations were detected to be 3087 $3766 \mu \mathrm{g} / \mathrm{mL}$ with the average value of $3369 \mu \mathrm{g} / \mathrm{mL}$. The much higher obtained concentration could be attributed that phenol-sulfuric acid method detected the hydrolyzed glucose content which could not eliminate other interfering substances like oligosaccharide. Aniline blue fluorescent method was suitable for the determination of triple helical lentinan, demonstrating the applicability of this method in complexed lentinan contained products.

\section{Conclusion}

In summary, we have optimized an aniline blue fluorescent method to quantify the amount of bioactive lentinan with the triple helical conformation. The method

Table 1. Determination of lentinan concentration in health tonic solution using aniline blue fluorescent method $(\mathrm{n}=3)$.

\begin{tabular}{ccccc}
\hline $\begin{array}{c}\text { Added lentinan } \\
(\mu \mathrm{g} / \mathrm{mL})\end{array}$ & $\begin{array}{c}\text { Determinedlentinan } \\
(\mu \mathrm{g} / \mathrm{mL})\end{array}$ & Recovery $(\%)$ & $\begin{array}{c}\text { Average recovery } \\
(\%)\end{array}$ & Average RSD (\%) \\
\hline 0 & 5.13 & 100 & & \\
2.5 & 7.70 & 103 & 101 & 1.73 \\
5.0 & 10.2 & 101 & & \\
7.5 & 12.5 & 98.9 & & \\
\hline
\end{tabular}


is fast, simple and convenient in operation. The detection limit of this method is lower than or at least comparable to other polysaccharide detection methods. Importantly, the protocol exhibits excellent selectivity for the determination of bioactive lentinan with triple helical structure over other saccharides. The method has been successfully used for the detection of lentinan in health tonic solution, which demonstrate the method has great practicability for quality control of lentinan related healthy products. It should also be noted that the excitation and emission of wavelength of this method is located in the visible region (400 $600 \mathrm{~nm}$ ) which may suffer autofluorescence in more complexed biological system. This study should substantially broaden the perspective for further manufacturing a near-infrared $(600-900 \mathrm{~nm})$ fluorescence probe to achieve precise determination of lentinan in related products.

\section{Conflicts of Interest}

The authors declare no conflicts of interest regarding the publication of this paper.

\section{References}

[1] Chihara, G., Maeda, Y., Hamuro, J., Sasaki, T. and Fukuoka, F. (1969) Inhibition of Mouse Sarcoma 180 by Polysaccharides from Lentinus edodes (Berk.) Sing. Nature, 222, 687-688. https://doi.org/10.1038/222687a0

[2] Chihara, G., Hamuro, J., Maeda, Y.Y., Arai, Y. and Fukuoka, F. (1970) Fractionation and Purification of the Polysaccharides with Marked Antitumor Activity, Especially Lentinan, from Lentinus edodes (Berk.) Sing, (an Edible Mushroom). Cancer Research, 30, 2776-2781.

[3] Hamuro, J. and Chihara, G. (1985) A Cell-Oriented Immunopotentiator: Its Experimental and Clinical Applications and Possible Mechanism of Immune Modulation. In: Fehichel, R.N. and Chirigos, M.A., Eds., Immune Modulation Agents and Their Mechanisms, Marcel Dekker, New York, 409-435.

[4] Kenji, I., Takae, K. and Takafumi, A. (2013) The Use of Lentinan for Treating Gastric Cancer. Anti-Cancer Agents in Medicinal Chemistry 13, 681-688. https://doi.org/10.2174/1871520611313050002

[5] Maeda, Y.Y. and Chihara, G. (1971) Lentinan, a New Immuno-Accelerator of Cell-Mediated Responses, Nature, 229, 634-636. https://doi.org/10.1038/229634a0

[6] Wasser, S.P. (2002) Medicinal Mushrooms as a Source of Antitumor and Immunomodulating Polysaccharides. Applied Microbiology and Biotechnology, 60, 258274. https://doi.org/10.1007/s00253-002-1076-7

[7] Mizuno, T. (1999) The Extraction and Development of Antitumor-Active Polysaccharides from Medicinal Mushrooms in Japan. International Journal of Medicinal Mushrooms, 1, 9-29. https://doi.org/10.1615/IntJMedMushrooms.v1.i1.20

[8] Oka, M., Yoshino, S., Hazama, S., Shimoda, K. and Suzuki, T. (1992) Immunological Analysis and Clinical Effects of Intraabdominal and Intrapleural Injection of Lentinan for Malignant Ascites and Pleural Effusion. Biotherapy, 5, 107-112. https://doi.org/10.1007/BF02171695

[9] Mueller, A., Raptis, J., Rice, P.J., Kalbfleisch, J.H., Stout, R.D., Ensley, H.E., Browder, W. and Williams, D.L. (2000) The Influence of Glucan Polymer Structure and Solution Conformation on Binding to $(1 \rightarrow 3)-\beta$-D-Glucan Receptors in a Human Monocyte-Like Cell Line. Glycobiology, 10, 339-346. 
https://doi.org/10.1093/glycob/10.4.339

[10] Sletmoen, M. and Stokke, B.T. (2008) Higher Order Structure of (1,3)- $\beta$-D-Glucans and Its Influence on Their Biological Activities and Complexation Abilities. Biopolymers, 89, 310-321. https://doi.org/10.1002/bip.20920

[11] Sasaki, T. and Takasuka, N. (1976) Further Study of the Structure of Lentinan, an Anti-Tumor Polysaccharide from Lentinus edodes. Carbohydrate Research, 47, 99-104. https://doi.org/10.1016/S0008-6215(00)83552-1

[12] Saito, H., Ohki, T., Takasuka, N. and Sasaki, T. (1977) A 13C-N.M.R.-Spectral Study of a Gel-Forming, Branched (1,3)- $\beta$-D-Glucan, (Lentinan) from Lentinus edodes, and Its Acid-Degraded Fractions. Structure, and Dependence of Conformation on the Molecular Weight. Carbohydrate Research, 58, 293-305.

https://doi.org/10.1016/S0008-6215(00)84356-6

[13] Saito, H., Ohki, T. and Sasaki, T. (1979) A ${ }^{13}$ C-Nuclear Magnetic Resonance Study of Polysaccharide Gels. Molecular Architecture in the Gels Consisting of Fungal, Branched (1,3)- $\beta$-D-Glucans (Lentinan and Schizophyllan) as Manifested by Conformational Changes Induced by Sodium Hydroxide. Carbohydrate Research, 74, 227-240. https://doi.org/10.1016/S0008-6215(00)84779-5

[14] Mizuno, T. (1995) Shiitake, Lentinus edodes. Functional Properties for Medicinal and Food Purposes. Food Reviews International, 11, 109-128. https://doi.org/10.1080/87559129509541022

[15] Bluhm, T. and Sarko, A. (1977) The Triple Helical Structure of Lentinan, a Linear $\beta$-(1-3)-D-Glucan. Canadian Journal of Chemistry, 55, 293-299. https://doi.org/10.1139/v77-044

[16] Hamuro, J., Maeda, Y.Y., Arai, Y., Fukuoka, F. and Chihara, G. (1971) The Significance of the Higher Structure of the Polysaccharides Lentinan and Pachymaran with Regard to Their Antitumour Activity. Chemico-Biological Interactions, 3, 69-71. https://doi.org/10.1016/0009-2797(71)90026-3

[17] Zhang, L., Li, X., Xu, X. and Zeng, F. (2005) Correlation between Antitumor Activity, Molecular Weight, and Conformation of Lentinan. Carbohydrate Research, 340, 1515-1521. https://doi.org/10.1016/j.carres.2005.02.032

[18] Surenjav, U., Zhang, L., Xu, X., Zhang, X. and Zeng, F. (2006) Effects of Molecular Structure on Antitumor Activities of (1-3)- $\beta$-D-Glucans from Different Lentinus edodes. Carbohydrate Polymers, 63, 97-104.

https://doi.org/10.1016/j.carbpol.2005.08.011

[19] Dubois, M., Gilles, K.A., Hamilton, J.K., Rebers, P.A. and Smith, F. (1956) Colorimetric Method for Determination of Sugars and Related Substances. Analytical Chemistry 28, 350-356. https://doi.org/10.1021/ac60111a017

[20] Masuko, T., Minami, A., Iwasaki, N., Majima, T., Nishimura, S.I. and Lee, Y.C. (2005) Carbohydrate Analysis by a Phenol-Sulfuric Acid Method in Microplate Format. Analytical Biochemistry, 339, 69-72. https://doi.org/10.1016/j.ab.2004.12.001

[21] McCleary, B.V. and Codd, R. (1991) Measurement of (1-3),(1-4)- $\beta$-D-Glucan in Barley and Oats: A Streamlined Enzymatic Procedure. Journal of the Science of Food and Agriculture, 55, 303-312. https://doi.org/10.1002/jsfa.2740550215

[22] Mizono, M., Minato, K. and Tsuchida, H. (1996) Preparation and Specificity of Antibodies to an Anti-Tumor $\beta$-Glucan, Lentinan. IUBMB Life, 39, 679-685. https://doi.org/10.1080/15216549600201751

[23] Tanaka, S., Aketagawa, J., Takahashi, S. and Shibata, Y. (1991) Activation of a Limulus Coagulation Factor G by ( $1 \rightarrow 3)$-gӨD-Glucans. Carbohydrate Research, 218 , 
167-174. https://doi.org/10.1016/0008-6215(91)84095-V

[24] Fan, Y.S., Lu, M., Yu, X.A., He, M.L., Zhang, Y., Ma, X.N., Kou, J.P., Yu, B.Y. and Tian, J.W. (2019) Targeted Myocardial Hypoxia Imaging Using a NitroreductaseActivatable Near-Infrared Fluorescent Nanoprobe. Analytical Chemistry, 91, 65856592. https://doi.org/10.1021/acs.analchem.9b00298

[25] Takao, N., Bappaditya, R., Daisuke, Y., Junji, S., Tatsuhiro, Y. and Seiji, S. (2016) Emergent Molecular Recognition through Self-Assembly: Unexpected Selectivity for Hyaluronic Acid among Glycosaminoglycans. Angewandte Chemie International Edition, 55, 1-6.

[26] Smith, M.M. and McCully, M.E. (1978) A Critical Evaluation of the Specificity of Aniline Blue Induced Fluorescence. Protoplasma, 95, 229-254.

https://doi.org/10.1007/BF01294453

[27] Evans, N.A. and Hoyne, P.A. (1982) A Fluorochrome from Aniline Blue: Structure, Synthesis and Fluorescence Properties. Australian Journal of Chemistry, 35, 2571 2575. https://doi.org/10.1071/CH9822571

[28] Thistlethwaite, P., Porter, I. and Evans, N. (1986) Photophysics of the Aniline Blue Fluorophore: A Fluorescent Probe Showing Specificity toward (1,3)- $\beta$-D-Glucans. The Journal of Physical Chemistry, 90, 5058-5063. https://doi.org/10.1021/j100412a036

[29] Synytsya, A. and Novák, M. (2013) Structural Diversity of Fungal Glucans-Review. Carbohydrate Polymers, 92, 792-809. https://doi.org/10.1016/j.carbpol.2012.09.077

[30] Zhang, L.-N., Li, X.-L., Zhou, Q., Zhang, X.-F. and Chen, R.-Q. (2002) Transition from Triple Helix to Coil of Lentinan in Solution Measured by SEC, Viscometry, and ${ }^{13}$ C NMR. Polymer Journal, 34, 443-449. https://doi.org/10.1295/polymj.34.443

\section{Supporting Information}

Table S1. Determination of originallentinan concentration in health tonic solution using phenol-sulfuric acid method and aniline blue fluorescent method, respectively (Data are means $\pm S D, n=3$ ).

\begin{tabular}{ccccc}
\hline Samples & $\begin{array}{c}\text { Concentration determined } \\
\text { by phenol-sulfuric acid } \\
\text { method }(\mu \mathrm{g} / \mathrm{mL})\end{array}$ & $\begin{array}{c}\text { Average } \\
\text { concentration } \\
(\mu \mathrm{g} / \mathrm{mL})\end{array}$ & $\begin{array}{c}\text { Concentration } \\
\text { determined by aniline } \\
\text { blue method }(\mu \mathrm{g} / \mathrm{mL})\end{array}$ & $\begin{array}{c}\text { Average } \\
\text { concentration } \\
(\mu \mathrm{g} / \mathrm{mL})\end{array}$ \\
\hline 1 & $3528 \pm 11$ & $228 \pm 3$ & \\
2 & $3269 \pm 14$ & $206 \pm 2$ & \\
3 & $3108 \pm 23$ & & $203 \pm 2$ & 216 \\
4 & $3192 \pm 50$ & 3369 & $204 \pm 2$ & \\
5 & $3087 \pm 87$ & $197 \pm 2$ & \\
6 & $3367 \pm 28$ & $228 \pm 4$ & \\
7 & $3766 \pm 21$ & $236 \pm 2$ & \\
8 & $3647 \pm 10$ & & $231 \pm 1$ & \\
9 & $3409 \pm 35$ & & $207 \pm 5$ & \\
10 & $3318 \pm 43$ & & & \\
\hline
\end{tabular}

